\title{
SB365, Pulsatilla saponin D suppresses proliferation and induces apoptosis of pancreatic cancer cells
}

\author{
MI KWON SON*, KYUNG HEE JUNG*, HEE-SEUNG LEE, HYUNSEUNG LEE, \\ SOO JUNG KIM, HONG HUA YAN, YE-LIM RYU and SOON-SUN HONG \\ Department of Drug Development, College of Medicine, Inha University, \\ Sinheung-dong, Jung-gu, Incheon 400-712, Republic of Korea
}

Received March 8, 2013; Accepted May 22, 2013

DOI: $10.3892 /$ or.2013.2517

\begin{abstract}
Pulsatilla koreana has been used as a traditional medicine for the treatment of various diseases. The purpose of this study was to determine whether SB365, Pulsatilla saponin D isolated from the root of Pulsatilla koreana inhibits the progression of pancreatic cancer. We found that SB365 strongly suppressed the growth and proliferation of 5 human pancreatic cancer cell lines (MIAPaCa-2, BXPC-3, PANC-1, AsPC-1 and HPAC). The apoptotic effect of SB365 was demonstrated by increased levels of cleaved caspase- 3 and decreased Bcl-2 expression via mitochondrial membrane potential, as well as elevated numbers of terminal deoxynucleotidyl-transferasemediated dUTP nick end labeling (TUNEL)-positive apoptotic cells. SB365 was also found to exert an anti-angiogenic effect by decreasing the expression of HIF-1 $\alpha$ and VEGF, major factors of angiogenesis, which was confirmed by the suppression of tumor sphere formation of pancreatic cancer cells. An in vivo mouse xenograft study showed that SB365 significantly inhibited tumor growth through the induction of apoptosis and inhibition of angiogenesis with strong anticancer activity. Therefore, SB365 is a good candidate as a natural product for use in the treatment of pancreatic cancer.
\end{abstract}

\section{Introduction}

Pancreatic cancer is one of the most aggressive and lethal forms of cancer with an overall 5-year survival rate of less than $5 \%$. It is currently the fourth cause of death from cancer in the Western world. Despite this fact, there has been little improvement in its prognosis over the past 20 years (1). The poor prognosis of pancreatic cancer can be attributed to the

Correspondence to: Professor Soon-Sun Hong, Department of Drug Development, College of Medicine, Inha University, 3-ga, Sinheung-dong, Jung-gu, Incheon 400-712, Republic of Korea E-mail: hongs@inha.ac.kr

"Contributed equally

Key words: Pulsatilla saponin D, SB365, pancreatic cancer, apoptosis, angiogenesis lack of early diagnosis, the aggressive biological behavior of the tumor and the insensitivity to most therapies including chemotherapy, radiotherapy and immunotherapy (2). Moreover, few patients have the chance to undergo radical surgery because of its poor diagnosis and those patients with unresectable pancreatic cancer usually survive for no more than 3-5 months, despite systemic treatment (3). Currently, gemcitabine remains the most effectvie agent available for the treatment of advanced pancreatic cancer, but the disease rapidly develops resistance to the drug. Thus, novel and efficacious strategies including low toxic agents that can overcome either the effect of gemcitabine or chemoresistance to drugs are urgently needed for the treatment for pancreatic cancer.

Non-toxic phytochemicals offer promising options for effective chemotherapeutic strategies for various types of cancer. Some studies support the concept that the consumption of bioactive dietary phytochemicals reduces the risk of pancreatic cancer $(4,5)$. Additionally, a number of studies have shown that various herbal extracts and compounds possess antitumor activities (6). One of these is Pulsatilla koreana which belongs to the family Ranunculaceae. The roots of this plant have been widely used in traditional medicine for the treatment of various diseases, including malaria and amoebic dysentery (7). This compound has also been reported to possess anti-inflammatory and anti-parasitic effects (8). This plant includes various effective components such as saponins, ranunculin, anemonin, protoanemonin and triterpenes (9). Specifically, there are 17 saponins in Pulsatilla koreana, among which, saponin D has been reported to demonstrate cytotoxicity against lung cancer cells $(10,11)$. Thus, Pulsatilla saponin D (hereafter designated as SB365) was selected from many types of saponins isolated from Pulsatilla koreana for further investigation. In the present study, we assessed the chemotherapeutic effect of SB365 in pancreatic cancer cells and conducted in vivo tumor xenograft studies. Our results showed that treatment with SB365 resulted in inhibition of cell growth/proliferation, angiogenesis, and induction of apoptosis in pancreatic cancer.

\section{Materials and methods}

Cells and materials. Human pancreatic cancer cell lines PANC-1, MIAPaCa-2, BXPC-3, AsPC-1 and HPAC were 
purchased from the American Type Culture Collection (AATC, Manassas, VA, USA). PANC-1 and MIAPaCa-2 cells were cultured in Dulbecco's modified Eagle's medium (DMEM). BXPC-3 and AsPC-1 cells were cultured in Roswell Park Memorial Institute (RPMI)-1640 medium, and HPAC cells were cultured in DMEM-F12 supplemented with $10 \%$ fetal bovine serum (FBS) and $1 \%$ penicillin/streptomycin. FBS and all other agents used in the cell culture studies were purchased from Invitrogen Life Technologies (Carlsbad, CA, USA). The cultures were maintained at $37^{\circ} \mathrm{C}$ in a $\mathrm{CO}_{2}$ incubator with a controlled humidified atmosphere composed of $95 \%$ air and $5 \% \mathrm{CO}_{2} \cdot 3$-(4,5-Dimethylthiazol-2-yl)-2,5-diphenyl tetrazolium bromide (MTT) and proteinase K were purchased from Sigma-Aldrich (St. Louis, MO, USA).

Preparation of SB365. SB365 was isolated from the roots of Pulsatilla koreana, as previously described by Kim et al (11). Briefly, the powdered roots $(50 \mathrm{~g})$ were extracted 3 times using $50 \%$ aqueous ethanol $(500 \mathrm{ml})$, and the residue was then suspended in $300 \mathrm{ml}$ of acetone and centrifuged. The precipitate was suspended in water and filtered to remove the insoluble portion. Next the fraction was chromatographed using a Sephadex LH-20 column $(200 \mathrm{~g}, 60 \mathrm{x} 4 \mathrm{~cm})$ and solid phase high performance liquid chromatography (HPLC; solid phase; RP-C18, 250x10 mm, mobile phase; $\mathrm{MeOH}: \mathrm{H}_{2} \mathrm{O}$ (82:20) as the mobile phase, $210 \mathrm{~nm}, 1 \mathrm{ml} / \mathrm{min}$ ) to yield SB365, a saponin D. To generate a more exact analysis of the purified SB365, we also used mass spectrometry and nuclear magnetic resonance (NMR) spectroscopy.

Measurement of cell proliferation. Cell viability was measured using the MTT assay. Briefly, PANC-1, BXPC-3, MIAPaCa-2, AsPC-1 and HPAC pancreatic cancer cells were plated at a density of $1-3 \times 10^{4}$ cells/well in 96 -well plates and then incubated for $48 \mathrm{~h}$. The media were then removed, and the cells were treated with either dimethyl sulfoxide (DMSO), as a negative control, or various concentrations of SB365. The final concentration of DMSO in the media was $\leq 0.1 \%$ (v/v). After the cells were incubated for $48 \mathrm{~h}, 20 \mu \mathrm{l}$ of MTT solution ( $2 \mathrm{mg} / \mathrm{ml}$ ) was added to each well, and the cells were incubated for another $4 \mathrm{~h}$ at $37^{\circ} \mathrm{C}$. The formed formazan crystals were dissolved in DMSO (200 $\mu \mathrm{l} /$ well) with constant shaking for $5 \mathrm{~min}$. The plate was then read on a microplate reader at $540 \mathrm{~nm}$. Three replicate wells were used for each analysis. The median inhibitory concentration $\left(\mathrm{IC}_{50}\right.$, defined as the drug concentration at which cell growth was inhibited by $50 \%$ ) was assessed using the resulting dose-response curves.

Immunofluorescence microscopy. Pancreatic cancer cells (PANC-1 and BXPC-3) were plated on 18-mm cover glasses in DMEM or RPMI-1640 medium and incubated for $24 \mathrm{~h}$ so that $\sim 70 \%$ confluence was reached. The cells were then incubated in the presence or absence of $10 \mu \mathrm{M}$ SB365, washed twice with PBS, and fixed in an acetone:ethanol solution (1:2) for $10 \mathrm{~min}$ at $-20^{\circ} \mathrm{C}$. Next, cells were blocked in $1.5 \%$ horse serum in PBS for $30 \mathrm{~min}$ at room temperature, and then incubated overnight at $4{ }^{\circ} \mathrm{C}$ with the primary antibody (1:50) in a humidified chamber. After washing twice with PBS, the cells were incubated with mouse fluorescein-labeled secondary antibody (1:100; Dianova, Hanburg, Germany) for $1 \mathrm{~h}$ at $37^{\circ} \mathrm{C}$. The cells were also stained with 4,6-diamidino-2-phenylindole (DAPI) to visualize the nuclei. The slides were then washed twice with PBS and covered with DABCO (Sigma-Aldrich) before being viewed with a confocal laser scanning microscope (Olympus, Tokyo, Japan).

DAPI staining and terminal deoxynucleotidyl-transferasemediated dUTP nick end labeling (TUNEL) assay. PANC-1 pancreatic cancer cells were plated onto 18 -mm cover glasses in DMEM medium and grown to $\sim 70 \%$ confluence for $24 \mathrm{~h}$. The cells were then treated with SB365 at a dose of $10 \mu \mathrm{M}$ for $24 \mathrm{~h}$. They were fixed in ice-cold $1 \%$ paraformaldehyde, washed with PBS and then stained with $2 \mu \mathrm{g} / \mathrm{ml}$ of DAPI for $20 \mathrm{~min}$ at $37^{\circ} \mathrm{C}$. The stained cells were examined for fluorescence of nuclear fragmentation. TUNEL was performed using the TUNEL kit (Millipore, Billerica, MA, USA).

\section{Measurement of mitochondrial membrane potential. BXPC-3} pancreatic cancer cells were plated on 18 - $\mathrm{mm}$ cover glasses in RPMI-1640 medium and incubated for $24 \mathrm{~h}$ so that $~ 70 \%$ confluence was reached. The cells were then incubated in the presence or absence of SB365 $(10 \mu \mathrm{M})$ for $6 \mathrm{~h}$ after which they were incubated with $5 \mu \mathrm{M} \mathrm{JC}-1$ fluorescence dye for $20 \mathrm{~min}$ in a $\mathrm{CO}_{2}$ incubator. The slides were then washed twice with PBS and covered with DABCO before being viewed with a confocal laser scanning microscope.

Analysis of cytochrome c localization. BXPC-3 cells were plated on 18-mm cover glasses in RPMI-1640 medium and incubated for $24 \mathrm{~h}$ so that $\sim 70 \%$ confluence was reached. The cells were then treated with SB365 $(10 \mu \mathrm{M})$ for $6 \mathrm{~h}$ and washed twice with PBS, and fixed in an acetic acid:ethanol solution (1:2) for $5 \mathrm{~min}$ at room temperature. Cells were incubated overnight at $4^{\circ} \mathrm{C}$ with cytochrome $c$ antibody (1:20; Santa Cruz Biotechnology, Inc., Santa Cruz, CA, USA). After washing twice with PBS, the cells were incubated with mouse fluorescein-labeled secondary antibody (1:50) containing $100 \mathrm{nM}$ of a mitochondrion-specific dye (MitoTracker ${ }^{\circledR}$ Red FM; Molecular Probes Inc., Eugene, OR, USA) for $45 \mathrm{~min}$ at $37^{\circ} \mathrm{C}$. The cells were also stained with DAPI to visualize the nuclei. The slides were then washed twice with PBS and covered with DABCO before viewing with a confocal laser scanning microscope.

Western blotting. BXPC-3 cells were washed 3 times with ice-cold PBS before lysis using a buffer containing $1 \%$ Triton X-100, $1 \%$ Nonidet P-40, and the following protease and phosphatase inhibitors: aprotinin $(10 \mathrm{mg} / \mathrm{ml})$, leupeptin (10 mg/ml) (ICN Biomedicals, Asse-Relegem, Belgium), phenylmethylsulfonyl fluoride $(1.72 \mathrm{mM}), \mathrm{NaF}(100 \mathrm{mM})$, $\mathrm{NaVO}_{3}(500 \mathrm{mM})$ and $\mathrm{Na}_{4} \mathrm{P}_{2} \mathrm{O}_{7}(500 \mathrm{mg} / \mathrm{ml})$ (Sigma-Aldrich). Equal amounts of protein were separated using $10 \%$ sodium dodecyl sulfate-polyacrylamide gel electrophoresis and transferred onto nitrocellulose membranes. The protein transfer was then checked using Ponceau S staining solution (Sigma-Aldrich). Immunostaining of the blots was performed using the primary antibodies, followed by the secondary antibody-conjugated to horseradish peroxidase with detection using enhanced chemiluminescence reagent (Amersham Biosciences, Piscataway, NJ, USA). Primary antibodies were 
purchased as follows: Bcl-2 (Santa Cruz Biotechnology Inc.), $\beta$-actin (Abcam, Cambridge, UK), cleaved caspase-3 (Cell Signaling Technology, Inc., Beverly, MA, USA). The secondary antibodies were purchased from Amersham Biosciences. The bands were visualized with the ECL Plus system (Amersham Biosciences).

Tumor sphere assay. PANC-1 cells were seeded in a 24-well low adhesion plate (Corning Inc., Lowell, MA, USA) at a density of $1 \times 10^{4}$ cells/well and an initial volume of $500 \mathrm{ml}$ as described by Dontu et al (12). Spheres were grown in DMEM medium (Gibco-BRL, Carlsbad, CA, USA) supplemented with vitamin B27, $20 \mathrm{ng} / \mathrm{ml} \mathrm{FGF}$, and $20 \mathrm{ng} / \mathrm{ml}$ epidermal growth factor (EGF; R\&D Systems, Minneapolis, MN, USA) and $4 \mu \mathrm{g} / \mathrm{ml}$ heparin. Samples were subsequently treated with $10 \mu \mathrm{M}$ SB365. EGF and FGF were replenished every 3 days. Aside from the addition of heparin, cells were agitated daily to minimize clumping. Spheres were counted and photographed after 14 days of continuous growth factor and supplemental exposure. Spheres were monitored under the microscope daily to ensure that they were derived from single cells and that they did not become confluent during the experiment.

Tumor xenograft study. Male athymic BALB/c nude mice were obtained from Orient Bio. Animal Inc. (Seoul, Korea). Animal care and all experimental procedures were conducted in accordance with the approval and guidelines of the INHA Institutional Animal Care and Use Committee (INHA IACUC) of the Medical School of Inha University (approval ID: 090518-5). The animals were fed standard rat chow and tap water ad libitum, and maintained under a $12 \mathrm{~h}$ dark/light cycle at $21^{\circ} \mathrm{C}$. Male nude mice (6 weeks old, weighing 22-26 g) were randomly divided into 2 groups (control and SB365 $30 \mathrm{mg} / \mathrm{kg}$ ). PANC-1 cells were harvested and mixed with PBS ( $200 \mu \mathrm{l} / \mathrm{mouse}$ ) and then inoculated into one flank of each nude mouse $\left(5 \times 10^{6}\right.$ of PANC-1 cells). When the tumors had reached a volume of $\sim 50-100 \mathrm{~mm}^{3}$, mice were given an intraperitoneal injection of SB365 (30 mg/kg, treatment group) or the vehicle (200 $\mu$ l PBS, control group) for 37 days. The tumor dimensions were measured twice a week using a digital calliper and the tumor volume (V) was calculated using the formula: $\mathrm{V}=$ length $\mathrm{x}$ width ${ }^{2} \mathrm{x} 0.5$. At the end of the experiment, mice were sacrificed, and tumors were excised and weighed.

Immunohistochemistry. After being blocked with normal goat serum (Vector Laboratories, Burlingame, CA, USA) for $1 \mathrm{~h}$, frozen tissue sections were incubated for $1 \mathrm{~h}$ at room temperature in dilutions of 1:100 of PCNA, cleaved caspase-3, VEGF and CD34 antibodies. The sections were visualized by an avidin-biotin peroxidase complex solution using an ABC kit (Vector Laboratories). The sections were washed in PBS and developed with a diaminobenzidine tetrahydrochloride substrate for $15 \mathrm{~min}$ and then counterstained with hematoxylin.

Statistical analysis. Data are expressed as the means \pm SD. Statistical analysis was performed using ANOVA. A P $\leq 0.05$ was considered to indicate a statistically significant result. Statistical calculations were performed using SPSS software for Windows operating system (version 10.0; SPSS, Inc., Chicago, IL, USA).

\section{Results}

Effect of SB365 on the proliferation of human pancreatic cancer cells. To evaluate the anticancer properties of SB365, we first compared the cell growth of 5 pancreatic cancer cell lines (MIAPaCa-2, BXPC-3, PANC-1, AsPC-1 and HPAC cell lines) that had been treated with SB365. As shown in Fig. 1A, the cells were exposed to various concentrations $(0,1,2,5,10$ and $20 \mu \mathrm{M}$ ) of SB365 for $48 \mathrm{~h}$. The results revealed that SB365 treatment inhibited cell growth in a dose-dependent manner. SB365 induced a reduction in cell growth, starting at a dose of $1 \mu \mathrm{M}$ in pancreatic cancer cells and strongly inhibited up to $80 \%$ of cell growth at doses of 5 and $10 \mu \mathrm{M}$. To confirm the effect of SB365 on cell growth and proliferation, we identified expression of $\mathrm{Ki}-67$, a cell proliferation marker using a fluorescent imaging system. As shown in Fig. 1B, SB365 significantly decreased the number of Ki-67-positive cells, when compared with the control.

Effects of SB365 on the apoptotic cell death of pancreatic cancer cells. To identify the pro-apoptotic effect of SB365 on pancreatic cancer cells, we first conducted DAPI staining and TUNEL assay. As shown in Fig. 2A, the cells treated with $10 \mu \mathrm{M}$ of SB365 showed morphological features of apoptotic cells, such as bright nuclear condensation and perinuclear apoptotic bodies, upon DAPI staining. SB365-induced apoptosis was confirmed by detection of DNA fragmentation using TUNEL staining. To identify involvement of SB365 in changes in the mitochondrial membrane potential $\left(\Delta \Psi_{\mathrm{m}}\right)$, which are correlated with intrinsic apoptosis, we also performed JC-1 staining. As shown in Fig. 2B, control cells showed heterogeneous staining of the cytoplasm with both red and green fluorescence coexisting in the same cells. Consistent with mitochondrial localization, red fluorescence (corresponding to high mitochondrial membrane potential) was primarily found in granular structures distributed throughout the cytoplasm. Treatment of PANC-1 and BXPC-3 cells with SB365 decreased the red fluorescence and led to occurrence of frequent clusters of mitochondria. Treatment of SB365 induced marked changes in $\Delta \Psi_{\mathrm{m}}$ evidenced by the disappearance of red fluorescence or the increase in green fluorescence in most cells. In addition, we observed that SB365 led to increased cytochrome $c$ release along with a concomitant decrease in the-co-localization of cytochrome $c$ and mitochondria (Fig. 2C). Furthermore, SB365 increased the protein expression levels of cleaved caspase- 3 and decreased Bcl-2 expression in BXPC-3 pancreatic cancer cells (Fig. 2D). Collectively, these results indicate that SB365 induced apoptosis of pancreatic cancer cells.

Effects of SB365 on the expression of HIF-1 $\alpha / V E G F$ and tumor sphere formation of pancreatic cancer cells. Considering the importance of HIF-1 $\alpha$ and VEGF in hypoxia, we examined the effects of SB365 on the expression pattern of HIF-1 $\alpha$ and VEGF in BXPC-3 cells. BXPC-3 pancreatic cancer cells were treated with $\mathrm{SB} 365(10 \mu \mathrm{M})$ under mimicked hypoxia induced by treatment with $100 \mu \mathrm{M} \mathrm{CoCl}_{2}$ for $24 \mathrm{~h}$. As shown in Fig. 3A, the HIF-1 $\alpha$ and VEGF expression increased under hypoxic condition, whereas SB365 treatment inhibited the hypoxia-induced HIF-1 $\alpha$ and VEGF expression. Moreover, to test for the ability of pancreatic cancer cells to grow as tumor spheres in culture, PANC-1 cells were plated on ultra-low 
$\mathbf{A}$
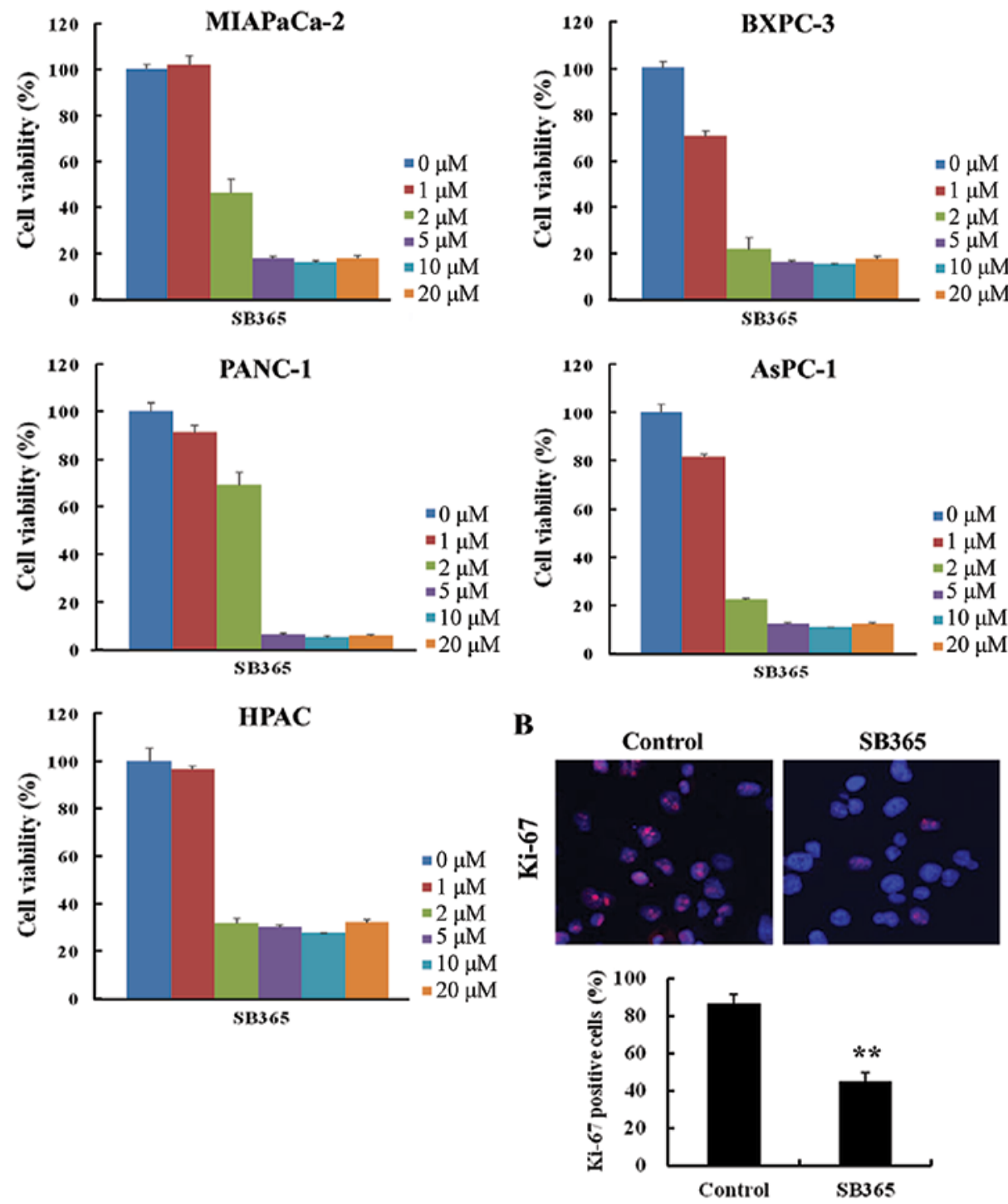

Figure 1. Effect of SB365 on the proliferation of human pancreatic cancer cells. (A) Cytotoxic effects of SB365. Viability of MIAPaCa-2, BXPC-3, PANC-1, AsPC-1 and HPAC pancreatic cancer cells was measured by MTT assay. Pancreatic cancer cells were seeded in 96-well culture plates. After incubation for $24 \mathrm{~h}$, the cells were treated with various concentrations of SB365 or with $0.1 \%$ dimethyl sulfoxide (DMSO) as a negative control. After incubation for $48 \mathrm{~h}$, the cells were subjected to the MTT assay. (B) Effect of SB365 on proliferation of PANC-1 cells was measured by Ki-67 staining, which was photographed at x200 magnification. Results are expressed as percent cell proliferation relative to the proliferation of the control. Data are represented as the means \pm SD from triplicate wells. ${ }^{* *} \mathrm{P}<0.01$ vs. control.

attachment plates in defined medium containing EGF, FGF and B27. After 2 weeks, tumor spheres consisting of 50-100 cells were observed, $\sim 50 \%$ of which had symmetrical ball-like structures (Fig. 3B). However, treatment of SB365 inhibited the formation of tumor spheres of pancreatic cancer cells in a dose-dependent manner.

Inhibition of pancreatic tumor growth by SB365 in a mouse xenograft model. Strong anticancer efficacy of SB365 led us to investigate the in vivo efficacy of SB365 against xenografts of PANC-1 pancreatic cancer cells in nude mice. As shown in Fig. 4A, SB365 suppressed tumor growth at a dose of $30 \mathrm{mg} / \mathrm{kg}$ for 37 days compared with the control group. SB365 administration resulted in a significant reduction in tumor volume $(55 \%)$ in nude mice. In addition, there was no difference in the body weight in the mouse groups treated with SB365 when compared with the control group, showing that SB365 exhibited little toxicity to mice at the curative dose (Fig. 4B). Consistent with this observation, the weight of tumors isolated from the SB365-treated mouse groups decreased significantly by $51 \%$ in the SB365 $30 \mathrm{mg} / \mathrm{kg}$ group, compared with the control (Fig. 4C, $\mathrm{P}<0.05$ ). Taken together, our results demonstrated that $\mathrm{SB} 365$ had strong antitumor efficacy against pancreatic cancer xenograft models.

Effects of SB365 on proliferation, angiogenesis and apoptosis in a xenograft model. Based on histopathological analysis using hematoxylin and eosin (H\&E) staining, we observed a greater degree of tumor apoptosis and necrosis in the SB365treated mouse group when compared to the control groups. 
A
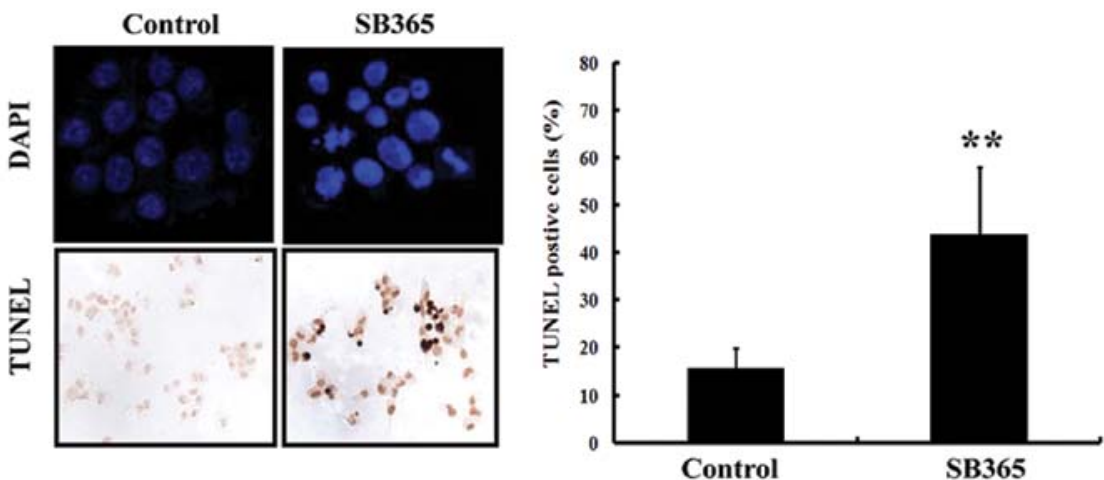

B
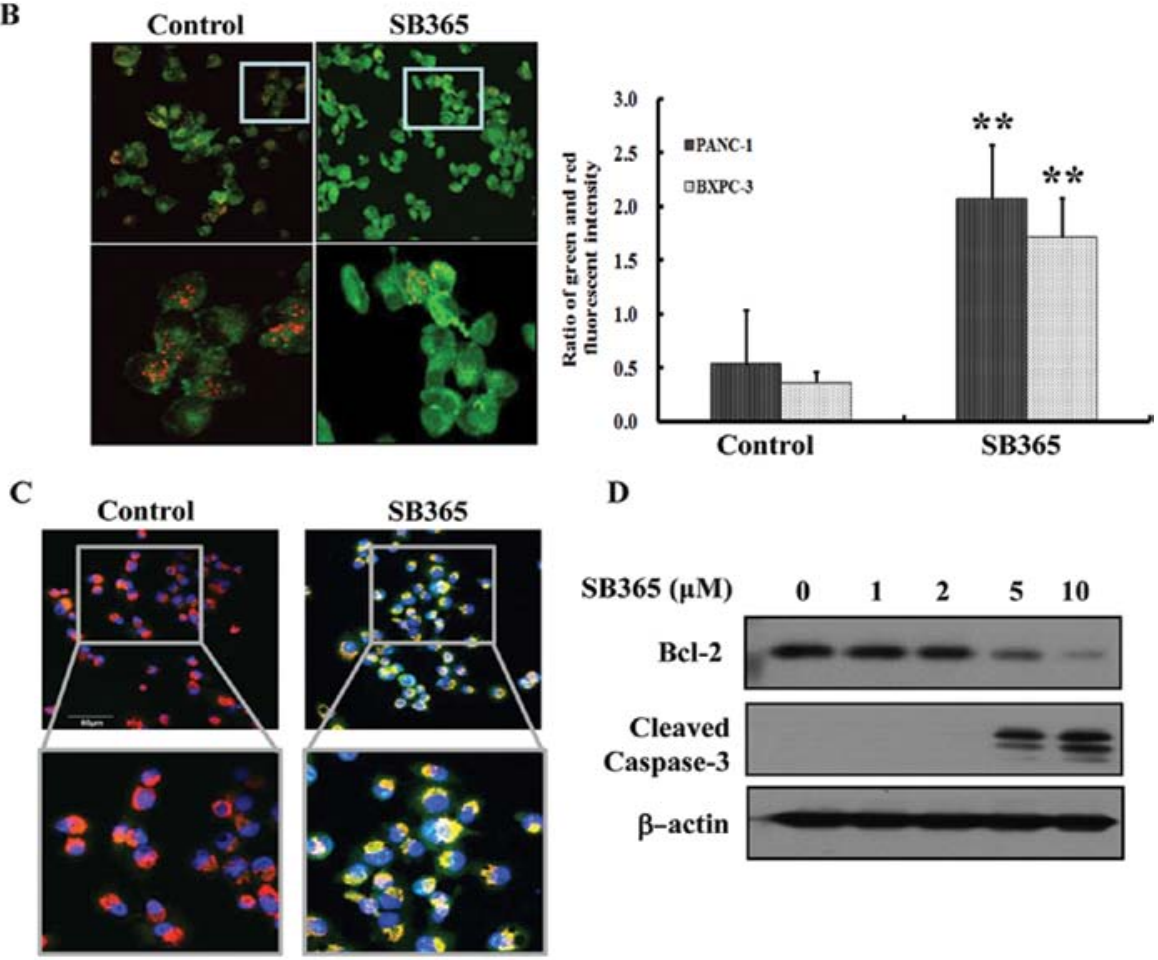

D
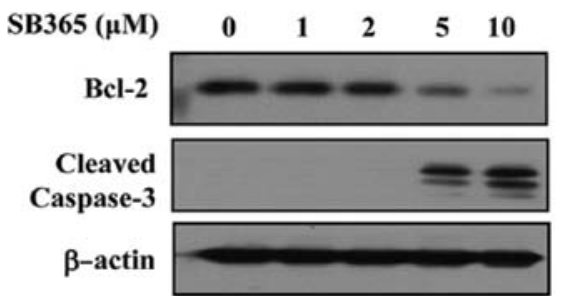

Figure 2. Effect of SB365 on the apoptosis of pancreatic cancer cells. (A) Induction of apoptosis in PANC-1 cells by SB365 (10 $\mu \mathrm{M})$ was determined by 4,6-diamidino-2-phenylindole (DAPI) and terminal deoxynucleotidyl-transferase-mediated dUTP nick end labeling (TUNEL) staining, which was photographed at $\mathrm{x} 400$ magnification. Data are expressed as the means \pm SD from the 3 experiments. ${ }^{* *} \mathrm{P}<0.01$ vs. control. (B) PANC-1 and BXPC-3 cells were treated with SB365 $(10 \mu \mathrm{M})$ for $6 \mathrm{~h}$, and the mitochondrial membrane potential was determined by JC-1 staining and analyzed using an LS 55 luminescence spectrometer, as well as photographed at x400 and x800 magnification (PANC-1 cells). The results (green/red ratio) are expressed as the percentage of cells treated with SB365. Data are expressed as the means \pm SD of triplicate wells. ${ }^{* *} \mathrm{P}<0.01$ vs. control. (C) After treatment with SB365 (10 $\left.\mu \mathrm{M}\right)$ for $6 \mathrm{~h}$, BXPC-3 cells were stained with anti-cytochrome $c$ antibody, MitoTracker and DAPI. The immunostained cells were analyzed under an Olympus confocal laser scanning microscope at x400 and x800 magnification (D) PANC-1 cells were treated with SB365 (0, 1, 2, 5 and $10 \mu \mathrm{M})$ for 48 h. Cell lysates were prepared and subjected to western blotting for Bcl-2, cleaved caspase-3 and $\beta$-actin.

Furthermore, SB365 decreased the expression of PCNA, a cell proliferation marker, as well as both VEGF and CD34 in the SB365-treated group compared with the control group. In addition, the apoptotic effect of SB365 on pancreatic cancer tumor tissues was identified by expression of cleaved caspase- 3 and DNA fragments by TUNEL assay. These results suggest that SB365 had an anti-angiogenic effect on pancreatic cancer xenografts (Fig. 5).

\section{Discussion}

Owing to the advanced stage of the disease and resistance to cytotoxic chemotherapeutic agents and radiation therapy, pancreatic cancer remains one of the most intractable human malignancies. In recent years, bioactive components from plant origin have obtained considerable attention as promising options for the development of effective strategies for the prevention or treatment of cancer. Accordingly, the discovery of active medicinal compounds from herbal/natural sources has provided alternative treatment choices for patients (13). Therefore, we isolated SB365, saponin D from Pulsatilla koreana and conducted a comprehensive analysis of the effect of SB365 on pancreatic cancer using both in vivo and in vitro models. In the present study, we report that SB365 significantly reduced cell growth and proliferation while inducing apoptosis in pancreatic cancer cells. This is the first report validating the anticancer properties of SB365 in both pancreatic cancer cells and a tumor xenograft animal model. 
A

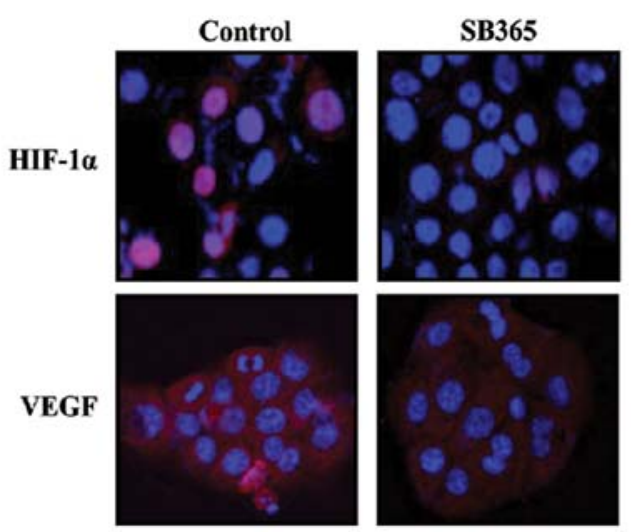

B

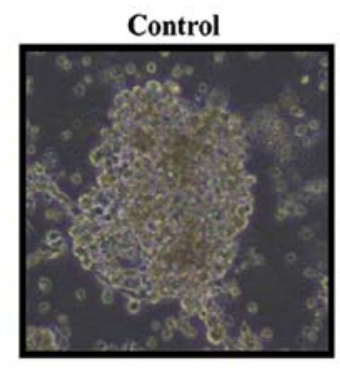

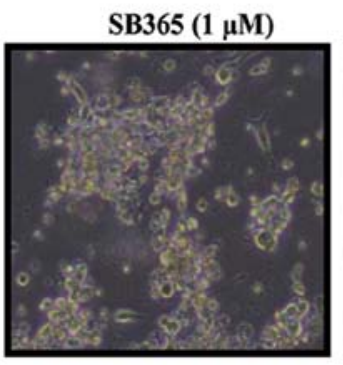

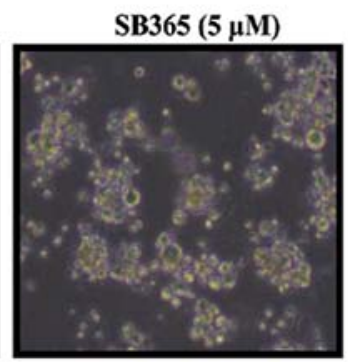

Figure 3. Effect of SB365 on the angiogenesis of pancreatic cancer cells. (A) Immunofluorescence imaging showing the expression of HIF-1 $\alpha$ and VEGF in hypoxic BXPC-3 cells (hypoxia induced with $100 \mu \mathrm{M} \mathrm{CoCl}_{2}$ ) treated with SB365 (10 $\left.\mu \mathrm{M}\right)$. Magnification, x800. (B) Typical PANC-1 cell tumor spheres generated from single cells after 14 days. Bright field micrographs showing tumor sphere formation in PANC-1 cells after treatment with SB365 (1 and $10 \mu \mathrm{M})$. Magnification x400.

Loss of cell proliferation and/or induction of apoptosis are two major mechanisms by which cancer cells are killed by chemotherapeutic agents (14). In this study, SB365 inhibited $50-80 \%$ of the growth of pancreatic cancer cells (MIAPaCa-1, BXPC-3, PANC-1, AsPC-1 and HPAC cells) at concentrations of 2-10 $\mu \mathrm{M}$, and the level of $\mathrm{Ki}-67-$ positive cells was also significantly decreased by SB365 treatment, indicating that SB365 has anti-proliferative effect. These results are in line with those of previous studies, in which many types of saponins were found to inhibit cell proliferation in various types of cancer cells (15-18).

While the effect of SB365 on proliferation likely contributes to its anticancer activity, it appears that pancreatic cancer cells are more sensitive to apoptosis induced by SB365. In fact, the $\mathrm{IC}_{50}$ values reflect the overall change in cell viability, which includes changes in both proliferation and apoptosis. Indeed, SB365 showed low $\mathrm{IC}_{50}$ values $(0.8-2 \mu \mathrm{M})$ in 5 pancreatic cancer cell lines. Therefore, we investigated whether SB365 had an apoptotic effect in pancreatic cancer. In our study, we observed that SB365 has the capacity to induce pancreatic cancer cells to undergo apoptosis. DNA fragmentation and nuclear chromatin condensation were demonstrated in pancreatic cancer cells treated with SB365. Moreover, the observation of caspase-3 activation and decreased Bcl-2 confirms the promotion of apoptosis by SB365. In addition, changes in the mitochondrial membrane potential are induced to create pores of mitochondria, which dissipates the transmembrane potential and leads to the release of cytochrome $c$ into the cytoplasm $(19,20)$, finally resulting in the induction of apoptosis. Therefore, to identify the effect of SB365 on mitochondrial membrane potential, we conducted JC-1 and cytochrome $c$ immunofluorescence staining. SB365 induced significant changes in mitochondrial membrane potential $\left(\Delta \Psi_{\mathrm{m}}\right)$ and increased cytochrome $c$ release along with a concomitant increase in co-localization of cytochrome $c$ with mitochondria. Collectively, these results indicate that SB365 induced an apoptotic effect by mitochondrial-mediated apoptotic molecules against pancreatic cancer cells.

Angiogenesis is a process that plays an important role in the growth and metastasis of solid tumors (21). The inhibition of angiogenic pathways is also an alternative for targeting cancer cell proliferation. In this process, VEGF is a potent inducer of angiogenesis and HIF- $1 \alpha$ is the major regulator of VEGF transcriptional activation $(22,23)$. In the present study, SB365 obviously decreased the expression of HIF-1 $\alpha$ and VEGF under hypoxia, showing that SB365 inhibited hypoxia-induced angiogenesis in pancreatic cancer. In our previous study, SB365 inhibited not only tube formation and migration of HUVECs but also the formation of neovessels in Matrigel plug (24). Based on these findings, we expected that the anti-angiogenic capacity of SB365 would accompany inhibition of metastasis, which is associated with tumor formation and migration. Indeed, bursts of angiogenic activity can be sufficient to initiate sustained tumor angiogenesis and metastasis (25). To grow beyond a small sphere, tumor cells must be able to invade across a barrier known as the basement membrane and into local tissue. At this point, they can also produce angiogenic factors such as VEGF that recruit 
A
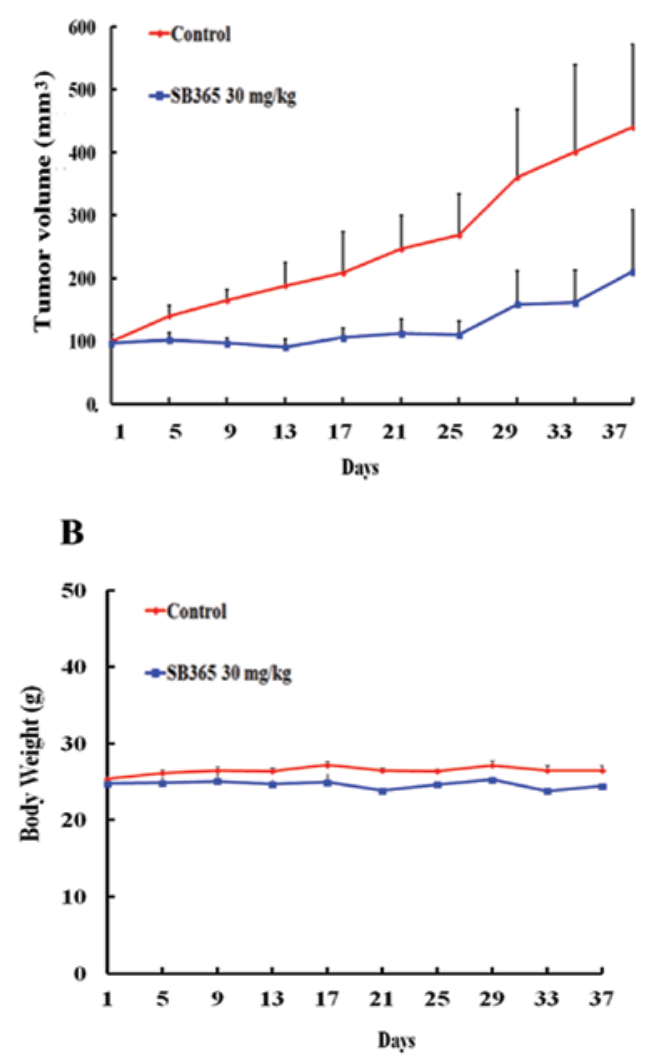

C
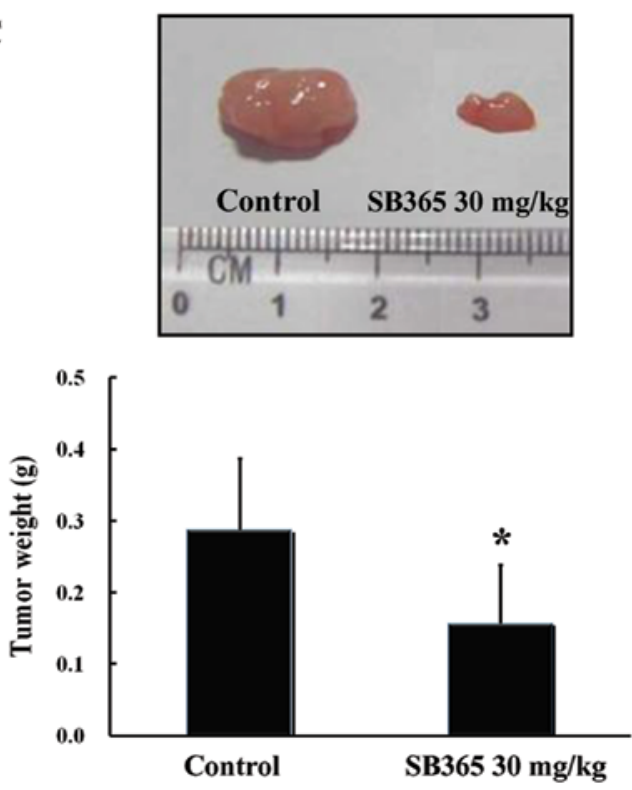

Figure 4. In vivo effect of SB365 in the pancreatic cancer mouse xenograft model. (A) Tumor growth of PANC-1 xenografts in nude mice. After the tumors reached $50-100 \mathrm{~mm}^{3}$ in size, the mice received an intraperitoneal administration of SB365 (30 mg/kg) 3 times a week for 37 days. (B) Average body weight of nude mice. (C) Tumor size and weight in PANC-1 mouse xenografts. Data are represented as the means $\pm \mathrm{SD}(\mathrm{n}=6) .{ }^{* *} \mathrm{P}<0.01$ vs. control.

new blood vessel formation (26). Moreover, when tumor cells are allowed to grow to a large size, these new vessels support tumor metastasis (27). Based on this knowledge, we evaluated whether SB365 inhibits pancreatic tumor sphere formation.

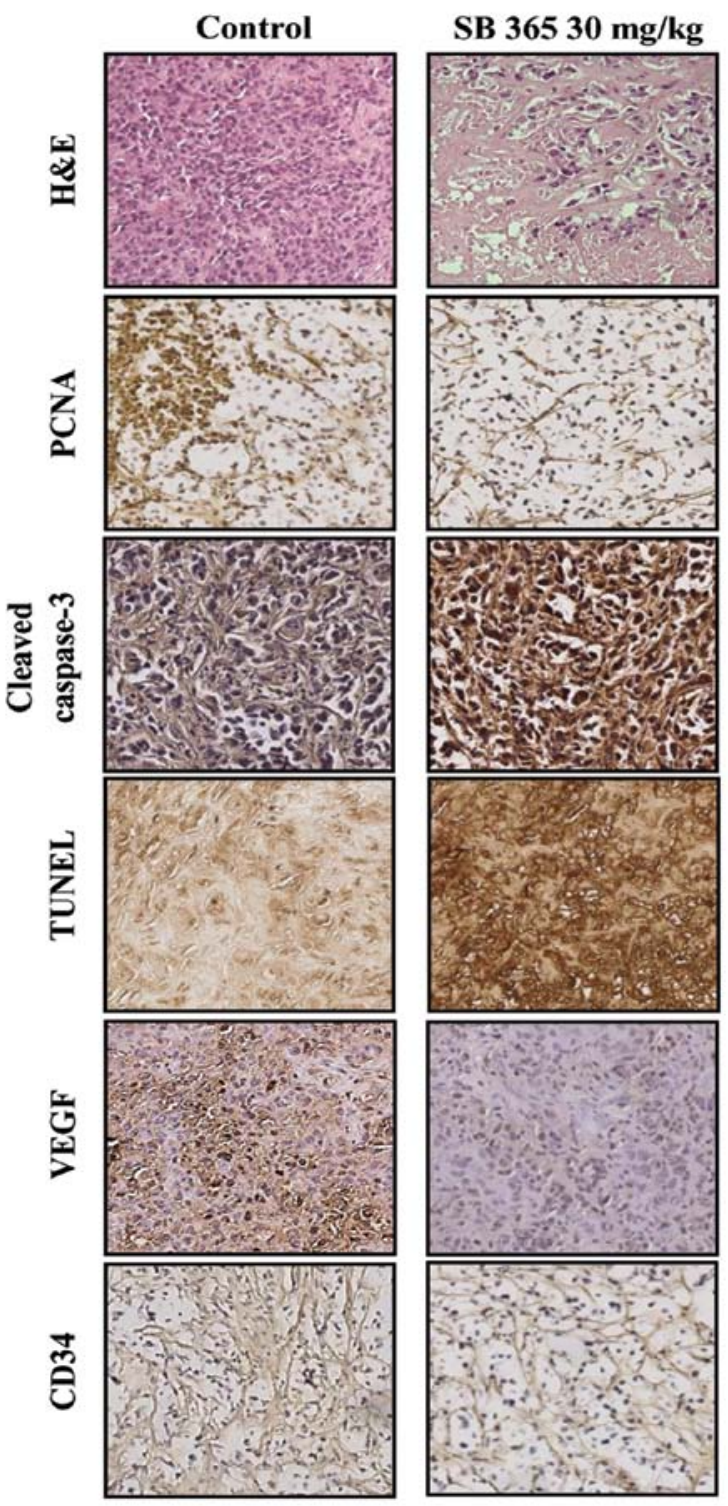

Figure 5. In vivo effect of SB365 on the proliferation, apoptosis and angiogenesis of PANC-1 xenografts. Tumors were excised and processed for immunostaining for PCNA, cleaved caspase-3, terminal deoxynucleotidyltransferase-mediated dUTP nick end labeling (TUNEL), VEGF and CD34 including hematoxylin and eosin (H\&E) staining. Magnification, $\mathrm{x} 400$.

Importantly, we found that SB365 obviously inhibited tumor sphere forming in a population of pancreatic cancer cells with tumor sphere-forming ability. These findings indicate that SB365 demonstrated potent anti-angiogenic effects along with the potential for inhibition of metastasis.

Most importantly, our in vitro results were corroborated in vivo in a pancreatic cancer xenograft model. Specifically, SB365 inhibited tumor growth in the mouse xenograft models. These data are in accordance with the decreased proliferation and angiogenesis documented by PCNA, and CD34 and VEGF immunostaining, respectively. Additionally, SB365 induced apoptosis as indicated by increased cleaved caspase- 3 and TUNEL staining in tumor tissues. These in vivo results are similar to our in vitro study, which further suggests that SB365 may be an applicable approach to enhance the antitumor activity against pancreatic cancer. 
In conclusion, the present study demonstrated that SB365 potentiated the anticancer effect against pancreatic cancer by inducing apoptosis as well as inhibiting cell growth/ proliferation and angiogenesis. Based on our findings, SB365 is a potential therapeutic agent against pancreatic cancer. However, further investigation to explore possible clinical uses of SB365 as a natural compound is needed in terms of therapeutic strategy.

\section{Acknowledgements}

This study was supported by the Korean Health Technology R\&D Project (A120266), the Ministry of Health and Welfare and the National Research Foundation of Korea (NRF) funded by the Ministry of Education, Science and Technology (NRF 2012-0002988, 2012R1A2A2A01045602, 2012R1A1A2043281) and the Inha University Grant.

\section{References}

1. Li D, Xie K, Wolff R and Abbruzzese JL: Pancreatic cancer Lancet 363: 1049-1057, 2004.

2. Saif MW: Adjuvant therapy of pancreatic cancer: beyond gemcitabine. Highlights from the '2011 ASCO Gastrointestinal Cancers Symposium'. San Francisco, CA, USA. January 20-22, 2011. JOP 12: 106-109, 2011.

3. Theissen G, Pardon B and Wagner R: A quantitative assessment for transcriptional pausing of DNA-dependent RNA polymerases in vitro. Anal Biochem 189: 254-261, 1990.

4. Block G, Patterson B and Subar A: Fruit, vegetables, and cancer prevention: a review of the epidemiological evidence. Nutr Cancer 18: 1-29, 1992.

5. Bueno de Mesquita HB, Maisonneuve P, Moerman CJ, Runia S and Boyle P: Life-time history of smoking and exocrine carcinoma of the pancreas: a population-based case-control study in The Netherlands. Int J Cancer 49: 816-822, 1991.

6. Cassileth BR: Alternative and complementary medicine. Separating the wheat from the chaff. Cancer 86: 1900-1902, 1999.

7. Bae K (ed). The Medicinal Plants of Korea. Kyu-Hak Press, Seoul, pp139, 1999.

8. Ye WC, Ou BX, Ji NN, Zhao SX, Ye T, McKervey MA and Stevenson P: Patensin, a saponin from Pulsatilla patens var. multifida. Phytochemistry 39: 937-939, 1995.

9. Ye WC, Ji NN, Zhao SX, Liu JH, Ye T, McKervey MA and Stevenson P: Triterpenoids from Pulsatilla chinensis. Phytochemistry 42: 799-802, 1996.

10. Bang SC, Lee JH, Song GY, Kim DH, Yoon MY and Ahn BZ: Antitumor activity of Pulsatilla koreana saponins and their structure-activity relationship. Chem Pharm Bull 53: 1451-1454, 2005.
11. Kim Y, Bang SC, Lee JH and Ahn BZ: Pulsatilla saponin D: the antitumor principle from Pulsatilla koreana. Arch Pharm Res 27: 915-918, 2004.

12. Dontu G, Abdallah WM, Foley JM, Jackson KW, Clarke MF, Kawamura MJ and Wicha MS: In vitro propagation and transcriptional profiling of human mammary stem/progenitor cells. Genes Dev 17: 1253-1270, 2003.

13. Smith M and Boon HS: Counseling cancer patients about herbal medicine. Patient Educ Couns 38: 109-120, 1999.

14. Li Y, Ahmed F, Ali S, Philip PA, Kucuk O and Sarkar FH: Inactivation of nuclear factor $\kappa \mathrm{B}$ by soy isoflavone genistein contributes to increased apoptosis induced by chemotherapeutic agents in human cancer cells. Cancer Res 65: 6934-6942, 2005.

15. Li F, Fernandez PP, Rajendran P, Hui KM and Sethi G: Diosgenin, a steroidal saponin, inhibits STAT3 signaling pathway leading to suppression of proliferation and chemosensitization of human hepatocellular carcinoma cells. Cancer Lett 292: 197-207, 2010.

16. Peng L, Zhou Y, Kong de Y and Zhang WD: Antitumor activities of dammarane triterpene saponins from Bacopa monniera. Phytother Res 24: 864-868, 2010.

17. Tin MM, Cho CH, Chan K, James AE and Ko JK: Astragalus saponins induce growth inhibition and apoptosis in human colon cancer cells and tumor xenograft. Carcinogenesis 28: 1347-1355, 2007.

18. Chen PS, Shih YW, Huang HC and Cheng HW: Diosgenin, a steroidal saponin, inhibits migration and invasion of human prostate cancer $\mathrm{PC}-3$ cells by reducing matrix metalloproteinases expression. PLoS One 6: e20164, 2011.

19. Petronilli V, Penzo D, Scorrano L, Bernardi P and Di Lisa F: The mitochondrial permeability transition, release of cytochrome $c$ and cell death. Correlation with the duration of pore openings in situ. J Biol Chem 276: 12030-12034, 2001.

20. Armstrong JS: Mitochondria: a target for cancer therapy. Br J Pharmacol 147: 239-248, 2006.

21. Folkman J: Angiogenesis. Annu Rev Med 57: 1-18, 2006.

22. Xia C, Meng Q, Cao Z, Shi X and Jiang BH: Regulation of angiogenesis and tumor growth by 110 alpha and AKT1 via VEGF expression. J Cell Physiol 209: 56-66, 2006.

23. Jiang BH, Rue E, Wang GL, Roe R and Semenza GL: Dimerization, DNA binding, and transactivation properties of hypoxia-inducible factor 1. J Biol Chem 271: 17771-17778, 1996.

24. Hong SW, Jung KH, Lee HS, Choi MJ, Son MK, Zheng HM and Hong SS: SB365 inhibits angiogenesis and induces apoptosis of hepatocellular carcinoma through modulation of PI3K/Akt/mTOR signaling pathway. Cancer Sci 103: 1929-1937, 2012.

25. Indraccolo S, Stievano L, Minuzzo S, Tosello V, Esposito G, Piovan E, Zamarchi R, Chieco-Bianchi L and Amadori A: Interruption of tumor dormancy by a transient angiogenic burst within the tumor microenvironment. Proc Natl Acad Sci USA 103: 4216-4221, 2006.

26. Hoeben A, Landuyt B, Highley MS, Wildiers H, Van Oosterom AT and De Bruijn EA: Vascular endothelial growth factor and angiogenesis. Pharmacol Rev 56: 549-580, 2004.

27. Alitalo A and Detmar M: Interaction of tumor cells and lymphatic vessels in cancer progression. Oncogene 31: 4499-4508, 2012. 